\title{
ChAKRA : The high resolution charged particle detector array at VECC
}

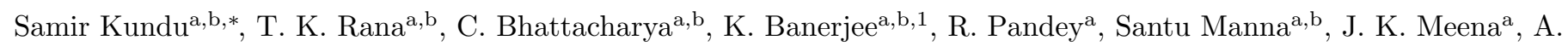

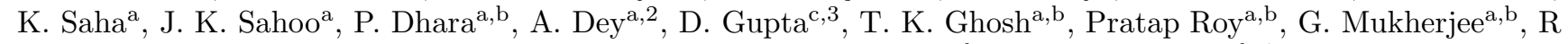 \\ Mandal Saha ${ }^{a}$, S. Roy ${ }^{a}$, S. R. Bajirao ${ }^{a}$, A. Sen ${ }^{a, b}$, S. Bhattacharya ${ }^{a, b, 4}$ \\ ${ }^{a}$ Variable Energy Cyclotron Centre, 1/AF, Bidhan Nagar, Kolkata - 700064 \\ ${ }^{b}$ Homi Bhabha National Institute, Training School Complex, Anushakti Nagar, Mumbai - 400 094, India \\ ${ }^{c}$ Department of Physics, Bose Institute, 93/1 Acharya Prafulla Chandra Road, Kolkata-700009, India
}

\begin{abstract}
'A large $4 \pi$ array of charged particle detectors has been developed at Variable Energy Cyclotron Centre to facilitate high resolution charged particle reaction and spectroscopy studies by detecting event-by-event the charged reaction products 'emitted in heavy ion reactions at energy $\sim 10-60 \mathrm{MeV} / \mathrm{A}$. The forward part $\left(\theta \sim \pm 7^{0}- \pm 45^{0}\right)$ of the array consists of 24 highly granular, high resolution charged particle telescopes, each of which is made by three layers [single sided silicon strip $(\Delta \mathrm{E})+$ double sided silicon strip $(\mathrm{E} / \Delta \mathrm{E})+\mathrm{CsI}(\mathrm{Tl})(\mathrm{E})]$ of detectors. The backward part of the array consists of $112 \mathrm{CsI}(\mathrm{Tl})$ detectors which are capable of detecting primarily the light charged particles $(\mathrm{Z} \leq 2)$ emitted in the angular range of $\theta \sim \pm 45^{0}- \pm 175^{0}$. The extreme forward part of the array $\left(\theta \sim \pm 3^{0}- \pm 7^{0}\right)$ is made up of 32 slow-fast plastic phoswich detectors that are capable of detecting light $(\mathrm{Z} \leq 2)$ and heavy charged particles $(3 \leq \mathrm{Z} \lesssim 20)$ as well as handling high count rates. The design, construction and characterization of the array has been described.
\end{abstract}

Keywords: Multidetector system; Silicon strip detector; CsI(Tl) detector; Plastic Phoswich detectors; Charged particle telescope; Pulse shape discrimination PACS: 29.40.Wk, 29.40.Mc, 29.30.Ep, 23.20.En

\section{Introduction}

The exploration of nuclear reaction dynamics is crucially dependent on the extent of information one can obtain about the reaction products; the more information one has about the reaction products, easier is the kinematic reconstruction of reaction events and deeper is the understanding of the reaction process. This is particularly important for heavy ion induced reactions at higher energies (above fermi energy), where multiplicity of reaction products is large. Event-by-event detection of maximum possible number of reaction products is necessary to enable faithful reconstruction of the events and characterise the reaction processes. Similarly, kinematic reconstruction technique also plays an important role for the study of structures of highly excited states (e.g., unbound resonances) in nuclei. To enable such measurements, one re"quires a large, granular array of detectors which is capable of detecting ideally all types of emitted charged particles

\footnotetext{
* Corresponding author.

Email addresses: skundu@vecc.gov.in (Samir Kundu), chandana@vecc.gov.in (C. Bhattacharya)

${ }^{1}$ Present address: Department of Nuclear Physics, Research School of Physics and Engineering, Australian National University, Canberra, ACT 2601, Australia

${ }^{2}$ Ex-research fellow.

${ }^{3}$ Some work done during tenure at VECC

${ }^{4}$ superannuated.
}

with sufficient precision over the whole solid angle, $4 \pi$. It is therefore not surprising that such detector arrays (e.g., INDRA [1], HIRA [2], CHIMERA 3], LASSA [4] etc.) serve as the main workhorse for reaction studies at all large accelerator centres across the world, and efforts are still continuing to build new (and/or upgrade old) detector arrays with higher granularity and better resolution (e.g. FAZIA [5])to probe more deeply into the nuclear reaction scenario.

The integrated experimental nuclear reaction programme based on the low $(\lesssim 10 \mathrm{MeV} / \mathrm{A})$ and intermediate energy ( $\sim 10-60 \mathrm{MeV} / \mathrm{A})$ heavy ions accelerated by the present K130 cyclotron and the upcoming K500 superconducting cyclotron at Variable Energy Cyclotron Centre (VECC), Kolkata, necessitated the development of one large, granular, high resolution charged particle detector array. The detector elements are required to have large dynamic range to cope up with experiments using both the accelerators. Moreover, the design should be flexible enough to enable either a part or whole of the detector system to be used in conjunction with other types of detector systems. With due consideration of the above, a Charged particle detector Array for Kinematic Reconstruction and Analysis (ChAKRA) has been developed at VECC, which is capable of detecting a wide range of emitted charged particles $(1 \leqslant Z \lesssim 20)$ over $\sim 4 \pi$ solid angle. The design details, fabrication and characterisation of various components of the detector array have 


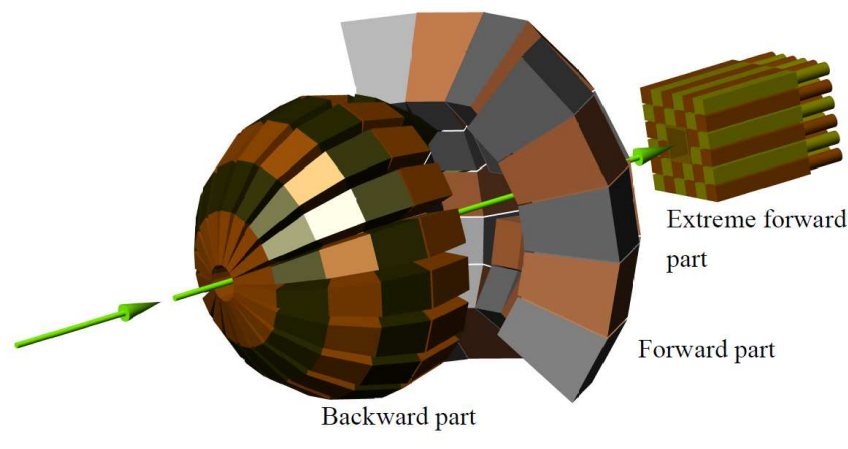

Figure 1: Schematic view of ChAKRA.

been reported in the present paper.

\section{Design details of the ChAKRA}

Depending upon the reaction kinematics, yield of different types of particles will vary with angles. Therefore, the type and granularity of the detectors should also vary with the angular range. According to that, the array has been configured in three independent blocks, all of which are sections of concentric spheres of different radii with the target position as the centre. A schematic view of the charged particle detector array is shown in Fig. 1. The coverage of each block is the surface area of the respective sphere spanned by two radial lines between $\theta_{\min } \leq \theta \leq \theta_{\max }$, where $\theta$ is the angle subtended by the radial line with the beam direction (see Fig. 1). The most important part of ChAKRA is the forward array block, which covers the angular range of $\theta \approx \pm 7^{0}- \pm 45^{0}$. Two other blocks are, the backward array (angular coverage : $\theta \approx \pm 45^{0}- \pm 175^{\circ}$ ), and the extreme forward array (angular coverage : $\left.\theta \approx \pm 3^{0}- \pm 7^{0}\right)$. The forward array consists of 24 charged particle telescopes, each of which is made up of 3 detector elements (Si(strip) - Si(strip) - CsI(Tl)). The backward array is made up of $112 \mathrm{CsI}(\mathrm{Tl})$ detectors of varying shapes and thicknesses. The extreme forward array consists of 32 plastic fast-slow phoswich detectors.

\subsection{Forward part}

The basic requirement of the forward part of the array is that it should be capable of measuring both position and energy as well as identifying all light charged particles (LCP : $Z=1,2)$ and intermediate mass fragments (IMF) in the range $3 \leqslant Z \lesssim 20$ emitted in the reaction. In addition, the array should also enable isotopic identification of all LCPs and IMFs at least up to $Z \lesssim 10$. To achieve these, single-sided Si strip detector (SSSD) of thickness $50 \mu \mathrm{m}$ has been used as the outermost (facing the beam) $\Delta \mathrm{E}$ detector, and double-sided Si strip detector(DSSD) of thickness $\sim 500 \mu \mathrm{m} / 1 \mathrm{~mm}$ has been used as E detector in all 24 telescopes. Typical energy resolution of these detectors was $\lesssim 1 \%$. To take care of energetic LCPs and lighter IMFs which do not stop in the Si-detectors, an extra layer

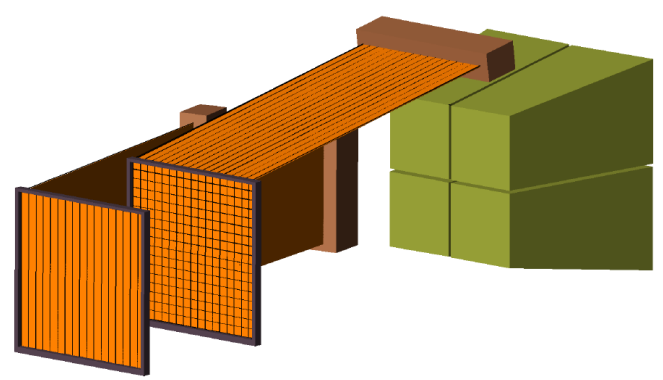

Figure 2: 3D schematic diagram of charged particle telescope used in the forward array. The left most detector is SSSD of thickness $\sim$ $50 \mu \mathrm{m}$, middle one is DSSD of thickness $\sim 500 \mu \mathrm{m}$ or $\sim 1000 \mu \mathrm{m}$ and right most detectors is an assembly os $4 \mathrm{CsI}(\mathrm{Tl})$ detectors of thickness $6 \mathrm{~cm}$.

of $\mathrm{CsI}(\mathrm{Tl})$ detector of thickness $6 \mathrm{~cm}$ was added behind the DSSD. The thickness of the CsI(Tl) detector was chosen to stop the highest energy light particles $(\sim 150 \mathrm{MeV}$ proton) expected in reactions at medium beam energies $(\approx$ $60 \mathrm{MeV} / \mathrm{A}$ ) from K500 superconducting cyclotron. Active area of each strip detector was $5 \times 5 \mathrm{~cm}^{2}$ with individual strip dimension of $3 \times 50 \mathrm{~mm}^{2}$. SSSD has 16 strips in front (junction) side and DSSD has 16 strips on both sides in mutually orthogonal direction as shown in Fig. 2, Each pixel in DSSD (size : $3 \times 3 \mathrm{~mm}^{2}$ ) provides an angular resolution of $\sim 0.8^{0}$ when kept at a distance of $20 \mathrm{~cm}$ from the target centre. Four $\mathrm{CsI}(\mathrm{Tl})$ detectors (each of front surface area $2.5 \times 2.5 \mathrm{~cm}^{2}$ and thickness $6 \mathrm{~cm}$ ) have been placed behind the thicker strip detector to complete each telescope assembly. $6 \mathrm{~cm} \mathrm{CsI(Tl)} \mathrm{itself} \mathrm{can} \mathrm{stop} \mathrm{proton} \mathrm{with} \mathrm{maxi-}$ mum energy $\sim 145 \mathrm{MeV}$. Total active area of four $\mathrm{CsI}(\mathrm{Tl})$ will cover all the particles which are not stopped by DSSD. The configuration of one telescope is shown schematically in Fig. 2]

\subsubsection{Forward part Si-strip detectors}

The silicon strip detectors (SSSD and DSSD) used in the present array are ion-implanted, passivated devices obtained from M/s. Micronsemiconductors Ltd., UK [ 6 . Total dead layer in the detector is typically $\sim 0.6 \mu \mathrm{m}$ including the implantation depth of about $\sim 0.40 \mu \mathrm{m}$. Typical energy resolutions of the detectors (individual strip) are, $\sim$ $40 \mathrm{keV}$ for the $(50 \pm 5) \mu \mathrm{m}$ SSSD and $\sim 40 \mathrm{keV}(\sim 25 \mathrm{keV})$ for $500 \mu \mathrm{m}(1000 \mu \mathrm{m})$ DSSD, measured using $5.486 \mathrm{MeV}$ ${ }^{241} \mathrm{Am}-\alpha$ source. The frames of the detectors were custom designed (see Fig. 3) to fit into the present array structure to minimise the dead area (frame size) surrounding each silicon wafer. The frame is made from glass epoxy with total outer dimension $60 \mathrm{~mm} \times 60 \mathrm{~mm}$ [Fig. 3]. A slot of width $1.75 \mathrm{~mm}$ and depth $2.25 \mathrm{~mm}$ in the inner (top) sides of the frame [Fig. 3] helps to glue the silicon wafer on the frame as well as protect the wafer from other detectors when used in telescopic mode. The outer ridge has four through holes, one in each corner, which can be used to align the frame by dowel pins. Signals from the strips are taken out by kapton cables with 18 soft gold pads of width 


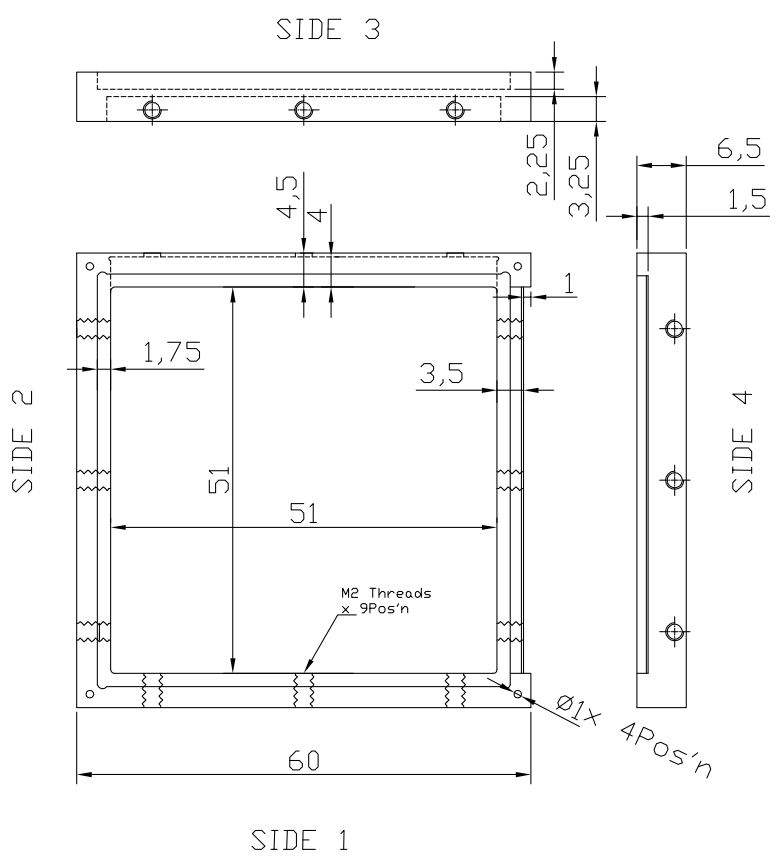

Figure 3: Design of the DSSD frame. All dimensions are in mm.

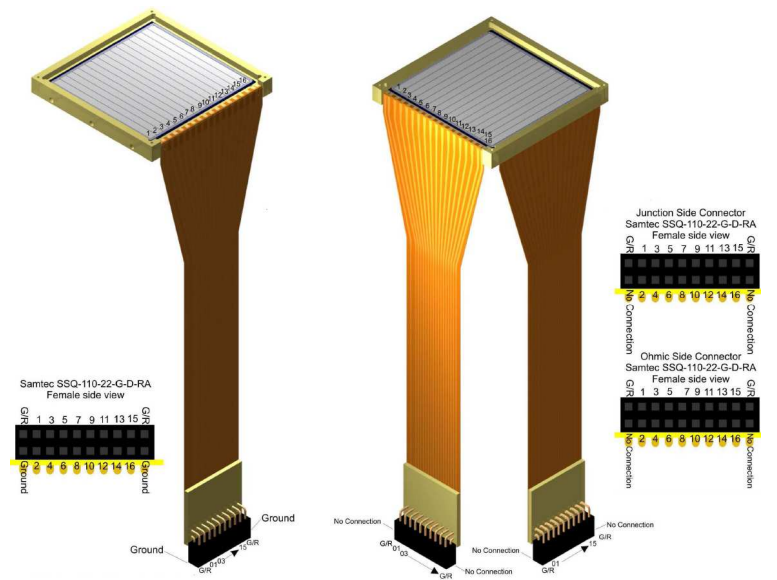

Figure 4: Strip detectors with kaptons.
$1.6 \mathrm{~mm}$ of each, the other end of which is connected to a flat ribbon connector(FRC) (SAMTEC SSQ-22-G-D-RA) (see Fig. 4). 16 pads are used for 16 signals connections and 2 for guard rings. The slots in side 4 ensure proper spacings for wire bonding of the front side strip with the kapton and its ( $\Delta \mathrm{E}$ detector) passage through the spacing between E detector's frame and housing wall. The slot in side 3 allows the kapton to be connected to backside strip (in DSSD). Both SSSD and DSSD frames are same in all respect except that there is no kapton in side 3 (Fig. 4) for SSSD.

\subsubsection{Forward part CsI(Tl) detector}

Thallium activated Cesium Iodide [CsI(Tl)] detectors constituted the third element of the forward array telescopes. All the CsI(Tl) detectors have been developed by $\mathrm{M} / \mathrm{s}$. Scionix Holland BV [7] as a complete assembly of CsI(Tl) crystal and photo diode (Hamamatsu S3204-08) with a charge sensitive preamplifier 7]. Custom made design of the detector has been done in association with $\mathrm{M} / \mathrm{s}$. Scionix Holland BV. The forward array has been designed in such a way that the front face of the first strip detector formed a part of the surface of a sphere of radius $\sim$ $20 \mathrm{~cm}$. Accordingly, the shape of the $\mathrm{CsI}(\mathrm{Tl})$ crystal has been decided such that the trajectories of the emitted particles were fully contained within the crystal. The design of the single detector, each of thickness $6 \mathrm{~cm}$, is shown in Fig. 5(A); the front and back faces are square shapes of dimensions $2.5 \mathrm{~cm} \times 2.5 \mathrm{~cm}$, and $3.5 \mathrm{~cm} \times 3.5 \mathrm{~cm}$, respectively. The thickness of $\mathrm{CsI}(\mathrm{Tl})$ is sufficient to stop a proton with energy $\sim 145 \mathrm{MeV}$. We have used an assembly of four such detectors in the third layer of the telescope; the segmentation provides better energy resolution and identification of highest energy lighter particles. The assembly of four $\mathrm{CsI}(\mathrm{Tl})$ forms a truncated pyramid of base area 7 $\mathrm{cm} \times 7 \mathrm{~cm}$ and its front face is having same area as the active area of the strip detectors as shown in Fig. 5(B). Each crystal has been wrapped in a special reflecting material covered with aluminized mylar of thickness $\sim 50 \mu \mathrm{m}$ on all sides except the front face. This will ensure better light collection and stop light leakage to adjacent detector. The front face has been covered with ultrathin $(\sim 1-2 \mu \mathrm{m})$ aluminized mylar sheet to allow the minimum dead layer for the incoming charged particles. Typical energy loss of $100 \mathrm{MeV} \alpha$ in $\sim 1-2 \mu \mathrm{m}$ aluminized mylar is $\sim 70-140 \mathrm{KeV}$ [8]. The crystals were coupled with photodiodes of active area $18 \mathrm{~mm} \times 18 \mathrm{~mm}$ (Hamamatsu S3204-08). A charge sensitive preamplifier [7] with gain of $\sim 4 \mathrm{~V} / \mathrm{pC}$ was directly coupled to the photodiode. A stack of four such detector are shown in Fig. 6]

\subsubsection{Forward part telescope housing}

The design of the telescope housing and its actual photograph after fabrication are shown in Fig. 7. A special hardened Aluminium alloy (6061-T6) has been used for fabrication of these housings. This alloy is easily machinable with high precision. The housings were designed in 
(A)
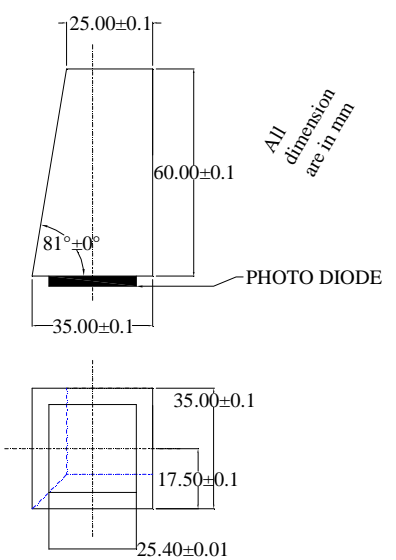

Figure 5: (A) Design of one $\mathrm{CsI}(\mathrm{Tl})$ detector and (B) assembly of four CsI crystal as used in the telescope.

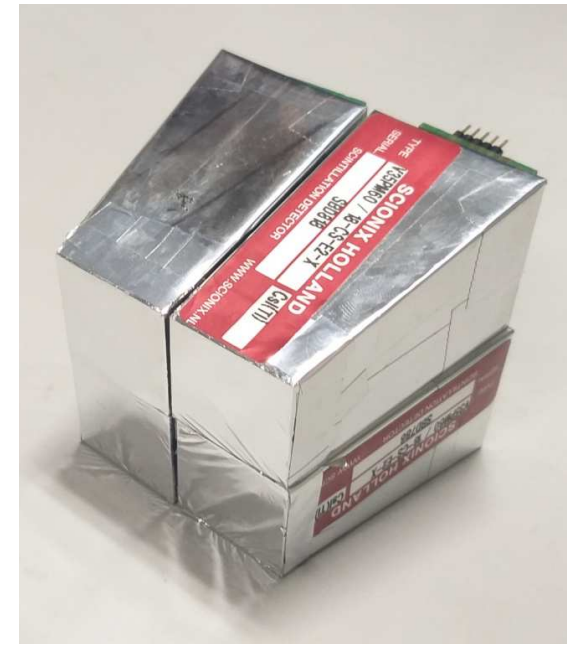

Figure 6: Assembly of four $\mathrm{CsI}(\mathrm{Tl})$ detectors for forward array telescope.
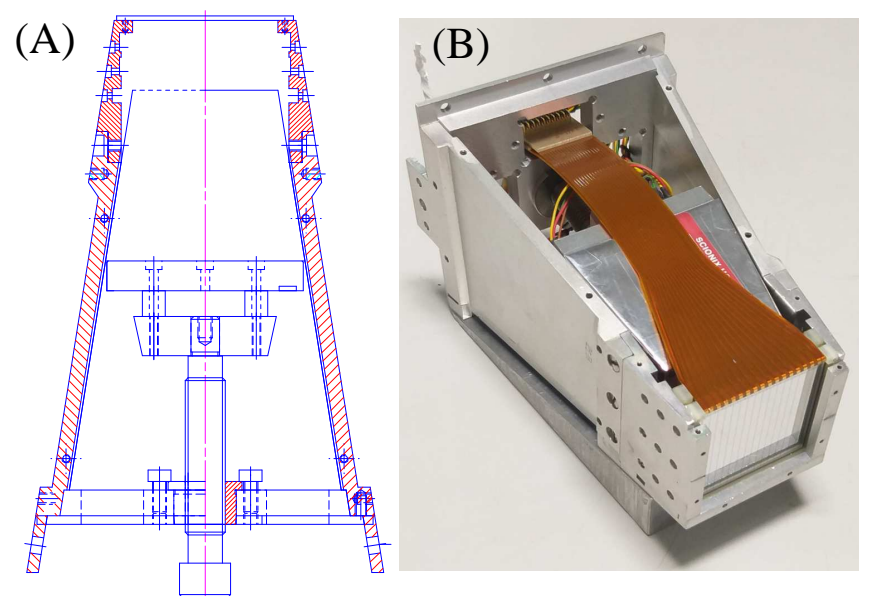

Figure 7: (A) Design of a telescope, and (B) photograph of the telescope with strip detectors and CsI detectors.

such a way that the telescope will contribute minimum dead area. The housing has two parts. In the front part, two strip detectors are kept in a fixed condition in two parallel slots. A provision is there to fix the strip detectors rigidly with the housing using screw. To keep the four $\mathrm{CsI}(\mathrm{Tl})$ detectors behind the strip detector (E), inner volume of the housing has been given a shape according to the overall shape of four CsI detector assembly, i.e., truncated pyramid shaped. In the inner sides of the housing there are slots of depth $0.8 \mathrm{~mm}$ and width $44 \mathrm{~mm}$ to take out the kapton of the strip detectors. To hold four CsI(Tl) detectors in proper position, there is an arrangement by a long threaded rod as shown by Fig. 7. By rotating the rod slowly, the detectors can be placed in proper position. The outer shape of the housings is such that when all the housings will be kept in the form of array, it will form a part sphere of radius $20 \mathrm{~cm}$. To attach all the housing in the array, a deep slot was kept outside of each four walls of the housings. A complete assembly of the housing is shown in Fig. 7 (right) with top open.

\subsubsection{Forward part support structure}

To mount the telescopes in a form of array, a support structure was developed. The telescopes have been arranged in 5 columns with five telescopes in each except in the middle column, where the central telescope has been removed for the passage of beam. Shapes of the columns are such that, all the front faces will touch the surface of an imaginary sphere of radius $20 \mathrm{~cm}$. In the same way, all the column have been placed so that front faces of all the telescopes together formed a part of the spherical surface of $20 \mathrm{~cm}$ radius spanning the angular range of $7^{0} \leq \theta \leq 45^{0}$. The whole array can be moved both vertically and horizontally to align it with the beam line axis. The complete assembly of 24 telescopes has been shown in Fig. 8. 


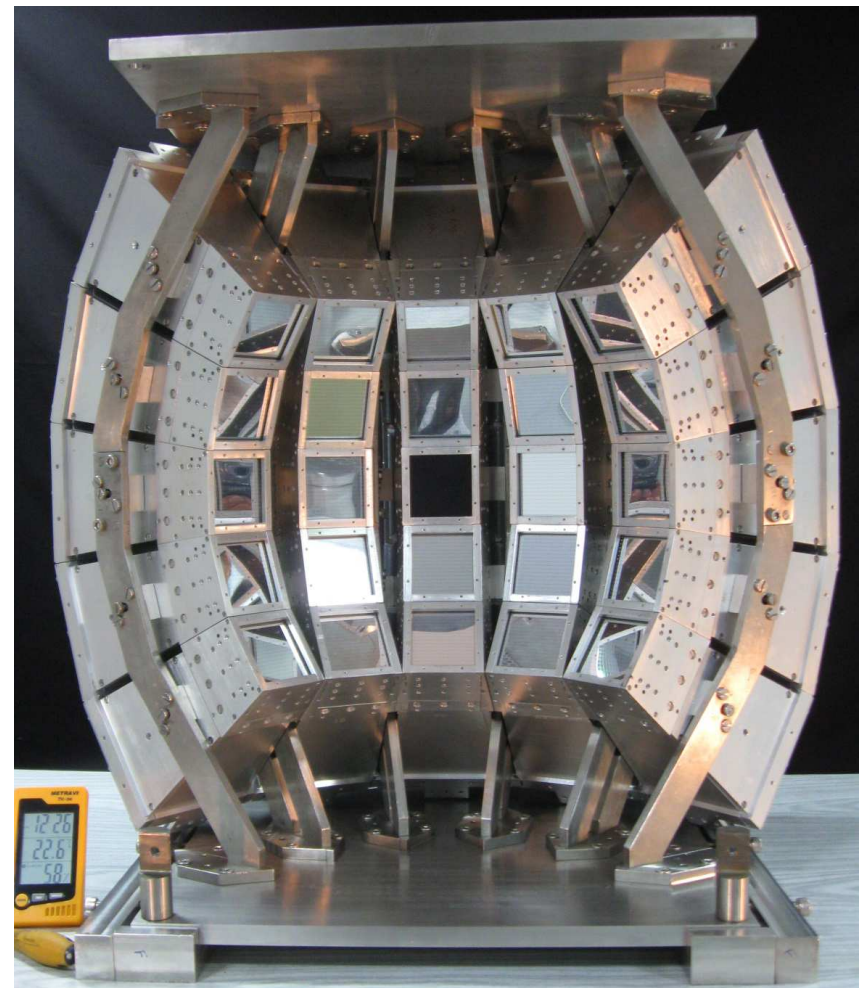

Figure 8: Forward array of charged particle telescopes.

\subsection{Backward array}

Since very few IMFs are emitted in the backward angular range (beyond the zone covered by the forward array), it has been decided to detect mainly the LCPs in the angular range $\theta=45^{0}-175^{\circ}$ using in backward part of ChAKRA. So, the backward part has been made up of $112 \mathrm{CsI}(\mathrm{Tl})$ detectors of various sizes as described below. Pulse shape discrimination (PSD) technique [9] has been used to identify the LCPs.

\subsubsection{Backward part detectors and support structure}

The shapes of the detectors of this array are such that the front faces of the detectors form the part of a spherical surface of radius $15 \mathrm{~cm}$ in the angular range $\theta=45^{\circ}-175^{\circ}$. The shapes and sizes of the detectors were optimised for minimum dead area and multihit probability. Each crystal, trapezoidal in shape, has been wrapped in a special reflecting material covered with $\sim 50 \mu \mathrm{m}$ thick aluminized mylar foil on all sides to prevent cross talk of scintillation light between the adjacent detectors. The front face has been covered with ultrathin $(\sim 1.5 \mu \mathrm{m})$ aluminized mylar. This allow the minimum dead layer for the incoming charged particles. Each detector was coupled, either directly or through light guide depending upon the thickness. It helps good light collection in the case of thinner detector (ring-3 to ring-6). All the crystals are coupled with photodiodes and vacuum compatible charged sensitive preamplifiers, forming an integrated detector assembly. The complete detector assembly, as per the design

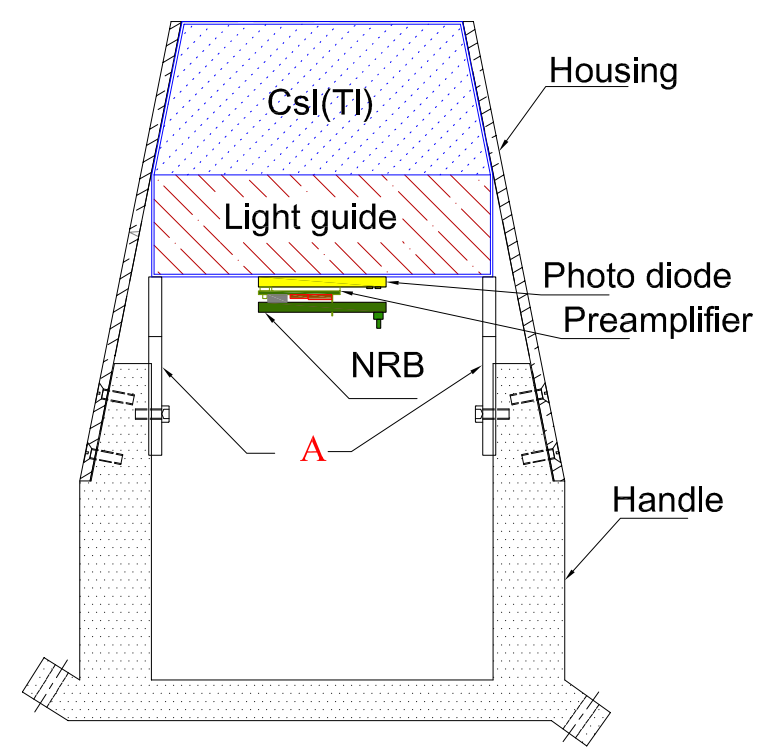

Figure 9: Schematic of Backward array $\mathrm{CsI}(\mathrm{Tl})$ detector with housing

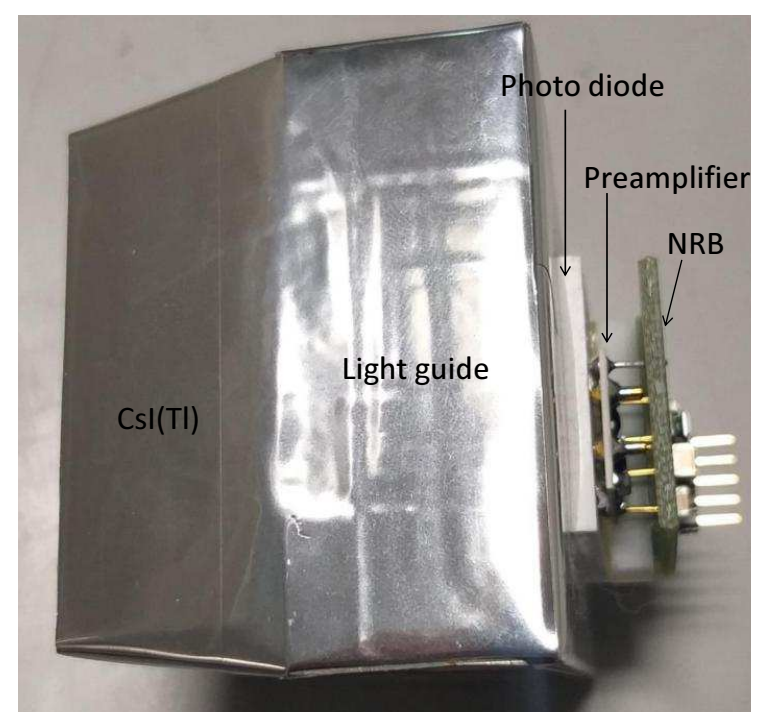

Figure 10: Typical backward array $\mathrm{CsI}(\mathrm{Tl})$ detector with preamplifier and noise reduction board (NRB). 
Table 1: Details of the $\mathrm{CsI}(\mathrm{Tl})$ detectors at different rings. $\mathrm{Rn} \rightarrow$ ring number, Th $\rightarrow$ detector thickness (in $\mathrm{cm}$ ), Type $\rightarrow$ Type of detector according to shape, $\mathrm{Q} \rightarrow$ Quantity of each type of detectors in any ring. The $\mathrm{A} \rightarrow$ Area $\left(\mathrm{cm}^{2}\right)$ of the front face of the detector, $\mathrm{PD}$ $\rightarrow$ Active area $\left(\mathrm{cm}^{2}\right)$ of photo diode and $\theta_{1}, \theta_{2}$ defines the angular width of each ring. Each detector covers an azimuthal width $\Delta \phi$ of $20^{0}$.

\begin{tabular}{cccccccc}
\hline Rn & Th & Type & Q & A & PD & $\theta_{1}$ & $\theta_{2}$ \\
\hline \multirow{2}{*}{1} & 4 & CsI-1 & 6 & 12.12 & 3.24 & 54.3 & 67.5 \\
2 & 4 & CsI-2 & 24 & 12.55 & 3.24 & 67.5 & 90 \\
& & CsI-2A & 2 & 12.23 & 3.24 & 67.5 & 73 \\
& & CsI-2B & 8 & 8.82 & 3.24 & 75.1 & 90.0 \\
3 & \multirow{2}{*}{3} & CsI-3 & 16 & 25.57 & 3.24 & 90.0 & 112.5 \\
& & CsI-3A & 2 & 12.23 & 3.24 & 100.4 & 112.5 \\
4 & 2 & CsI-4 & 18 & 21.23 & 3.24 & 112.5 & 135.0 \\
5 & 2 & CsI-3 & 18 & 13.08 & 2.00 & 135.0 & 157.5 \\
6 & 2 & CsI-4 & 18 & 2.87 & 1.00 & 157.5 & 170.4 \\
\hline
\end{tabular}

specifications, was fabricated by $\mathrm{M} / \mathrm{s}$. Scionix Holland $\mathrm{Bv}$ 7]. The size of the photodiode varied with the size of the detector. The design of a typical detector with housing and the photograph of a complete detector are shown in Figs. 9 and 10. In all, nine types of detectors of different shapes and thickness have been used in the array. The type of the detectors are named according to the ring number on which they are kept.

The design of the array support structure has been illustrated in Fig. 11. The backward array consists of six azimuthally symmetric rings. The details of the detectors and their placement in different rings is elaborated in Table 1. The ring-1 is partial and houses six detectors. Provision for inserting the target ladder has been kept between ring-2 and ring-3. All the other rings have eighteen detectors of a single type of detector in each ring (see Table 1). The design of the $\operatorname{array}($ Fig. 11) is such that each of the housing (Fig. 9) can be removed individually without disturbing the other detector and housings. At the end of the housing handle, two screws are there to attach the housing with the support structure. To fix the detector inside the housing two plates (marked with'A' in Fig. 9) are there which can be fixed on the handle with screw.

\subsection{Extreme forward array}

In the extreme forward angle (in the neighbourhood of grazing angle), detectors should be able to handle high counting rate and they should also be rugged against radiation damage due to high rate of bombardment. Plastic Scintillator is the quite suitable to handle such situation quite efficiently. So, the extreme forward array has been designed with 32 plastic phoswich detectors. Each phoswich detector was made up of $100 \mu \mathrm{m}$ (2ns) thin organic fast plastic scintillator (BC 408) ( $\Delta$ E-detector) and $10 \mathrm{~cm}$ slow (280ns) thick plastic scintillator (BC 444) (Edetector) as shown in Fig 13 schematically. Active area of

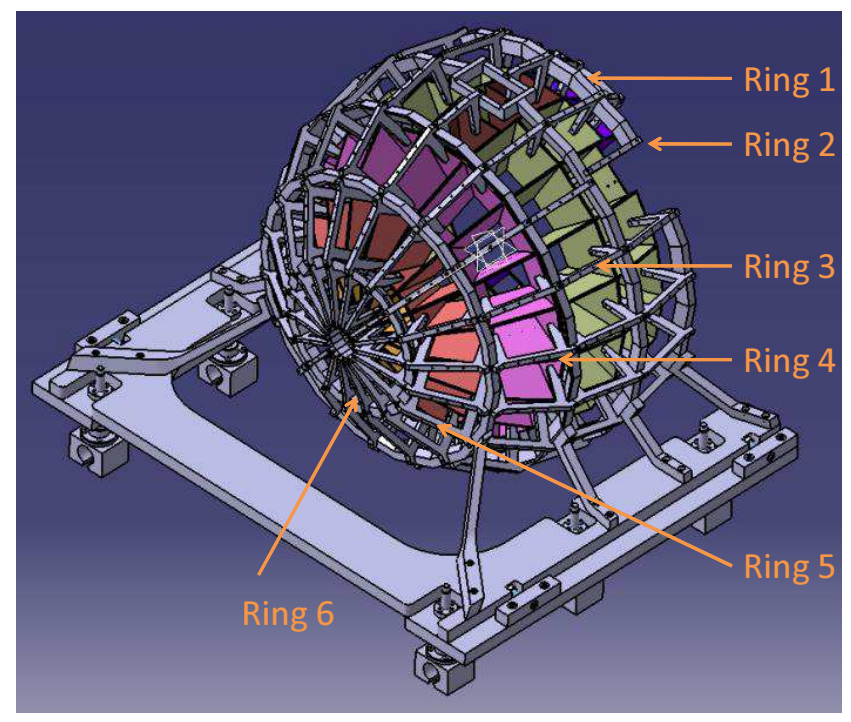

Figure 11: 3D CAD model of support structure and housings of backward part of ChAKRA

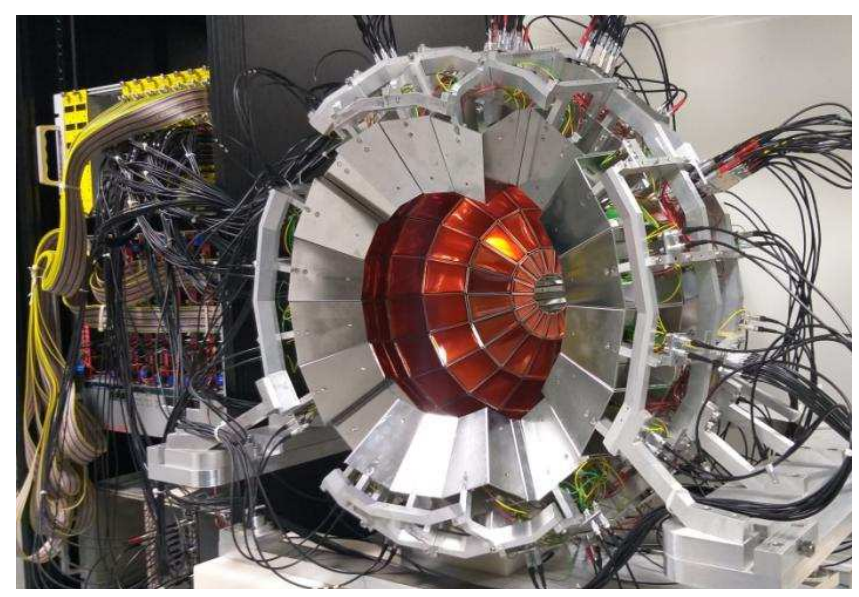

Figure 12: Backward part of ChAKRA with $112 \mathrm{CsI}(\mathrm{Tl})$ detectors and its electronics. 


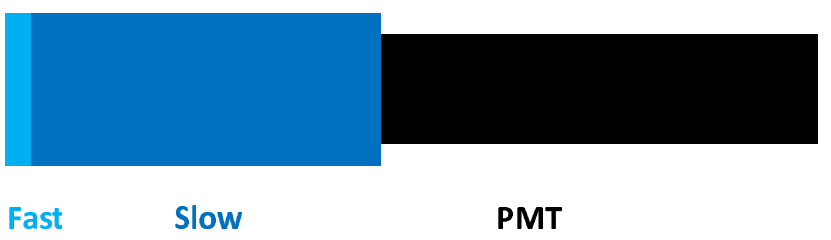

Figure 13: Schematic diagram of plastic phoswich detector with PMT.

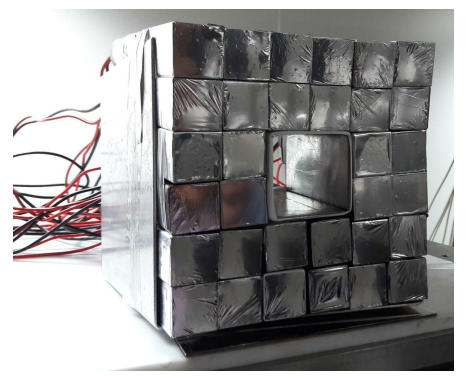

Figure 14: The array of 32 plastic phoswich detectors with a square shaped pipe with area of four detectors.

each detector is $20 \times 20 \mathrm{~mm}^{2}$ and it is coupled to a photomultiplier (Hamamatsu PMT model R6511) of $19 \mathrm{~mm}$ diameter. The array of these detectors is placed at $40 \mathrm{~cm}$ from the target which will cover an angular range of $\theta \sim 3^{0}$ to $7^{0}$. In the middle of the array, a square shaped pipe with area of four detectors, has been provided for transporting the beam to the beam dump. All plastic phoswich detectors of the array have been fabricated at VECC. Thick part of plastic used in the detector has been developed by M/s. Saint-Gobain Crystals [10] in proper shape. Thin plastic (BC 408) detectors of area $20 \times 20 \mathrm{~mm}^{2}$ have been prepared from large sheet of area $10 \times 10 \mathrm{~cm}^{2}$. Both this parts have been pasted with the optical cement, SC-500 7] and then painted with a white reflecting paint, SC 510 [7], to prevent scintillation light loss. The thin end of the detector has been covered with $1.5 \mu \mathrm{m}$ aluminized mylar foil and a photo multiplier tube has been optically coupled at the other end to make the phoswich detector. All sides of the plastic (except front) as well as the PMT joint were wrapped with a $50 \mu \mathrm{m}$ thick aluminized mylar foil to avoid scintillation light leak. The extreme forward array of plastic phoswitch detectors is shown in Fig 14 .

\subsection{Readout electronics and data acquisition system}

The readout of the array comprised of standard NIM/CAMAC front-end signal processing electronics followed by the VME Data Acquisition System (VMEDAQ). We have used 16 channel preamplifiers (MPR-16) and shaping amplifier cum constant fraction discriminator modules (MSCF-16)from M/s. mesytec GmbH \& Co. $K G$ [11] for the detectors used in the forward part of ChAKRA.The outputs of the shaping amplifiers are connected to 32 channel peak sensing VME ADC (Model CAEN V785). For backward part of ChAKRA, we used fast gate - slow gate charge integration method to identify the particle and measure of its energy. The output of the preamplifier coupled with $\mathrm{CsI}(\mathrm{Tl})$-photodiode was fed to the shaping amplifier cum leading edge discriminator module (MSCF-16) [1] specially designed by M/s. mesytec $\mathrm{GmbH} \& \mathrm{Co}$. KG for the present purpose. Charge integration was done using 32 channel VME QDC (Model CAEN V792). Double gate charge integration was also used for particle identification in the plastic phoshwich detectors of the forward part of ChAKRA. Here, the output of the PMT is directly amplified by a fast amplifier (CAEN N412) having two outputs in each channel . One output of each channel has been divided into two parts using a splitter circuit. The time trigger was generated using 16 channel CAMAC leading edge discriminator (LED) (CAEN C894). OR of two such LED are again ORed using 4 channel logic fan-in fan-out module having 4 input 4 output in each channel. Finally it goes to VME QDC (CAEN V792) through gate and delay generator (Ortec 8020)for fast gate- slow gate charge integration. Each ADC (QDC) module digitized the peak values (integrate charge) of all 32 channels simultaneously within $5.6 \mu$ s with 12 bit resolution and stores the data into FIFO. The VMEDAQ software reads the data in VME chain block transfer (CBLT) mode from multiple crates and builds the global events. The software parallelizes the operations like reading, event construction, storing and processing of the data using multiple threads in a producer-consumer model. The internal FIFO of the ADCs/QDCs and the multi-threaded parallel operations on a multi-core workstation are essential features to improve the dead time of the system. The current version of the data acquisition operates in common dead time mode. The common GATE signal is used for all the crates. A custom made sychronizer module is used to block the GATE signal, until all the ADC/QDC busy signals are withdrawn. This ensures the synchronization of the data acquisition by all the ADCs. The event correlation is achieved through hardware synchronization. Each event is marked with a GATE count value. This GATE count is used to correlate the events from different crates. The future upgradation of the VMEDAQ will also support the timestamping mechanism to correlate the events.

\section{Characterisation of ChAKRA}

Detailed offline and in-beam tests have been carried out to characterise various elements of ChAKRA. Initially, offline tests using radioactive sources have been done to check the basic parameters, like, energy resolution, thickness uniformity as well as uniformity of spatial response from the detectors for acceptability. In the next step, they have been tested in real in-beam experimental situation to test the performance. 


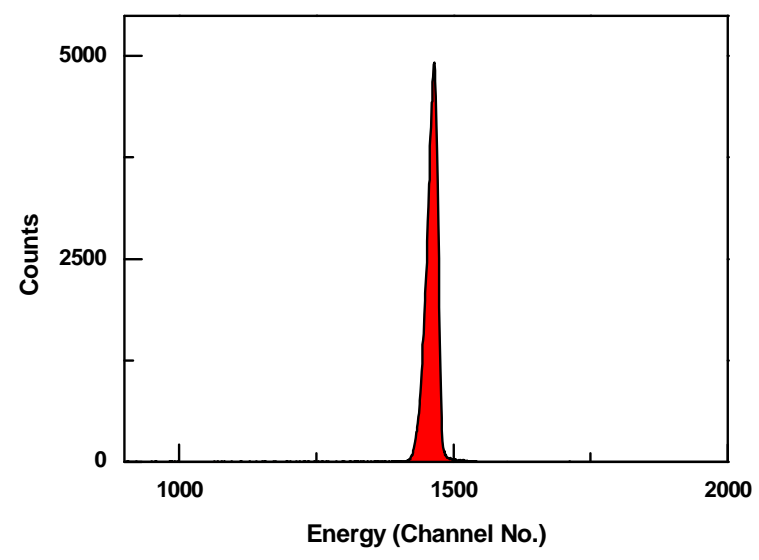

Figure 15: The energy spectrum of $\alpha$-particles emitted from ${ }^{241} \mathrm{Am}$ source obtained by an individual strip.

\subsection{Forward array}

\subsubsection{Characterization of silicon strip detectors}

The strip detectors have been tested in offline (wit sources) as well as in beam. Offline characterisation of individual strip has been done using a collimated pin${ }^{241} \mathrm{Am} 5.486 \mathrm{MeV} \alpha$-sources specially prepared in-h for this purpose. The pin source (diameter $1 \mathrm{~mm}$, c mated with a tube of length $5 \mathrm{~mm}$ ) was kept very clos $2 \mathrm{~mm}$ ) to the detector to irradiate the detector in a small area (circle of diameter $1.4 \mathrm{~mm}$ ) in a particular sition. The best energy resolutions of individual strip $50 \mu \mathrm{m}, 500 \mu \mathrm{m}$ and $1 \mathrm{~mm}$ detectors have been found $\mathrm{t}$ 45, 40 and $25 \mathrm{KeV}$, respectively. Typical energy spect of $\alpha$-source in one of the strips is shown in Fig. 15.

Thickness variation along a strip of the detector, ticularly in $50 \mu \mathrm{m}$ thin SSSD's, which are very pron thickness non-uniformity, results in poor isotopic id fication. So, it is very crucial to measure the thick non-uniformity, particularly for thin detectors. Ty] thickness non-uniformity of a thin $(50 \mu \mathrm{m}$ SSSD $) \mathrm{d}$ tor, estimated in an in-beam experiment, has been sh in Fig. 16. We have used $\alpha$ emitted in reaction 145 I ${ }^{20} \mathrm{Ne}+{ }^{12} \mathrm{C}$ using same method described in [4]. It been found that the variation in thickness along a str $\lesssim 3 \%$ of the average thickness.

\subsubsection{Characterisation of forward part CsI(Tl) detec}

The detailed characterisation of all $\mathrm{CsI}(\mathrm{Tl})$ detec have been done using ${ }^{241} \mathrm{Am} \alpha$-source. Typical en spectrum of the detectors has been displayed in Fig and the energy resolution was found to be $\sim 5 \%$. Dep ing upon the uniformity of the thallium doping and th ometry of the detector, there may be a non-uniformit the light output from different regions of a single CsI which may cause variation in isotopic resolution betr different pixels of DSSD and $\mathrm{CsI}(\mathrm{Tl})$ of the forward a telescope.

To measure the non-uniformity, a pin-type $\alpha$-source ..... been put at different positions very close $(2 \mathrm{~mm})$ to the

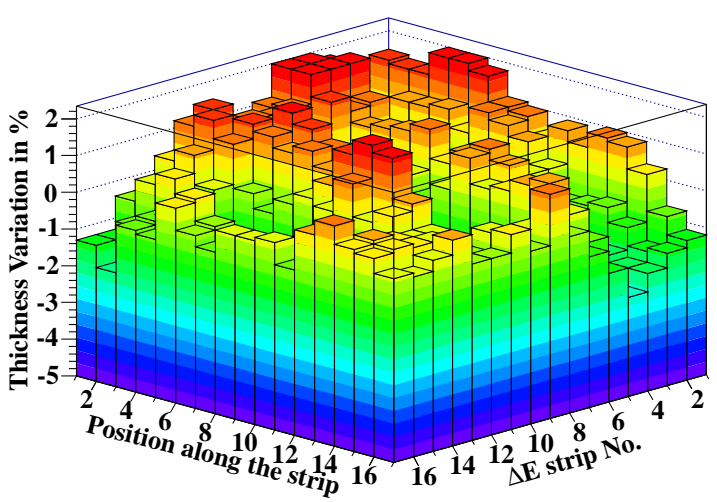

Figure 16: Non-uniformity in thickness along the strip in 16 different nositions for all strins of a. $50 \mathrm{~km}$ SSSD.

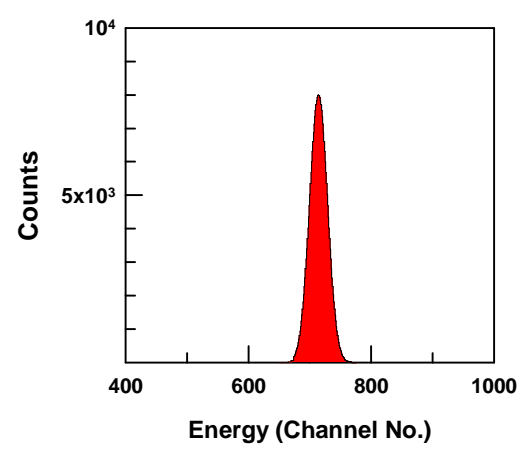

Figure 17: Spectrum of $\alpha$-particle emitted from ${ }^{241} \mathrm{Am}$ measured using $\mathrm{CsI}(\mathrm{Tl})$ detectors.

front face of the detector. Fig. 18 represents the front face (area $25 \mathrm{~mm} \times 25 \mathrm{~mm}$ ) of a $\mathrm{CsI}(\mathrm{Tl})$ detectors. Numbers are the peak positions of the $\alpha$-spectra in terms of ADC channel number at the centre of each block where the source has been kept during the test. With respect to the mean peak position, the variation was found to be $\sim \pm 0.49 \%$.

\subsubsection{Particle identification in forward part telescope}

After the fabrication of the forward telescopes, they have been tested in beam to check their performances, the particle identification in particular. In one test experiment described here, $193 \mathrm{MeV}{ }^{20} \mathrm{Ne}$ beam was incident on ${ }^{9} \mathrm{Be}$ target and the fragments were detected [12]. The telescope has been kept at $\sim 20 \mathrm{~cm}$. The angular resolution is $\sim \pm 0.8^{0}$. The $\Delta \mathrm{E}$-E spectrum of the emitted fragments obtained from first two layers [Si(SSSD)-Si(DSSD)] of the telescope is shown in Fig. 19. Clear isotopic separation of the fragments up to atomic number $\mathrm{Z}=10$ has been observed. Measurement and identification of LCPs, which punch through the two Si detector layers and deposit energy in $\mathrm{CsI}(\mathrm{Tl})$ detector at the back end of the telescope, require the usage of $\mathrm{Si}-\mathrm{CsI}(\mathrm{Tl})$ component of the telescope. A typical LCP study in the experiment of $60 \mathrm{MeV} \alpha$ on ${ }^{12} \mathrm{C}$ target is presented here, where the $\Delta \mathrm{E}$-E spectrum ob- 


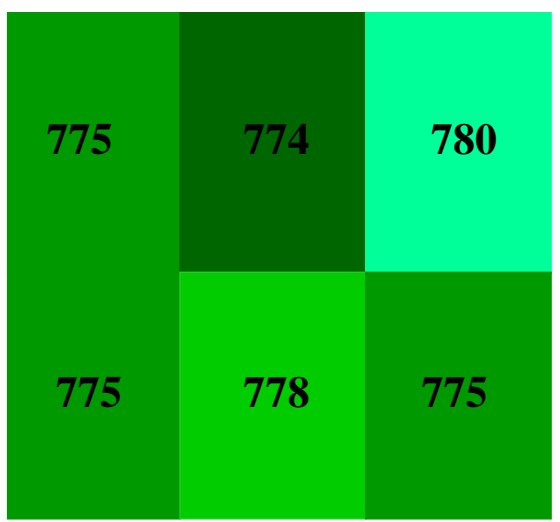

Figure 18: Non-uniformity in the light output with respect to the mean peak position (776). Numbers are the peak position of the $\alpha$-spectrum in terms of ADC channel number.

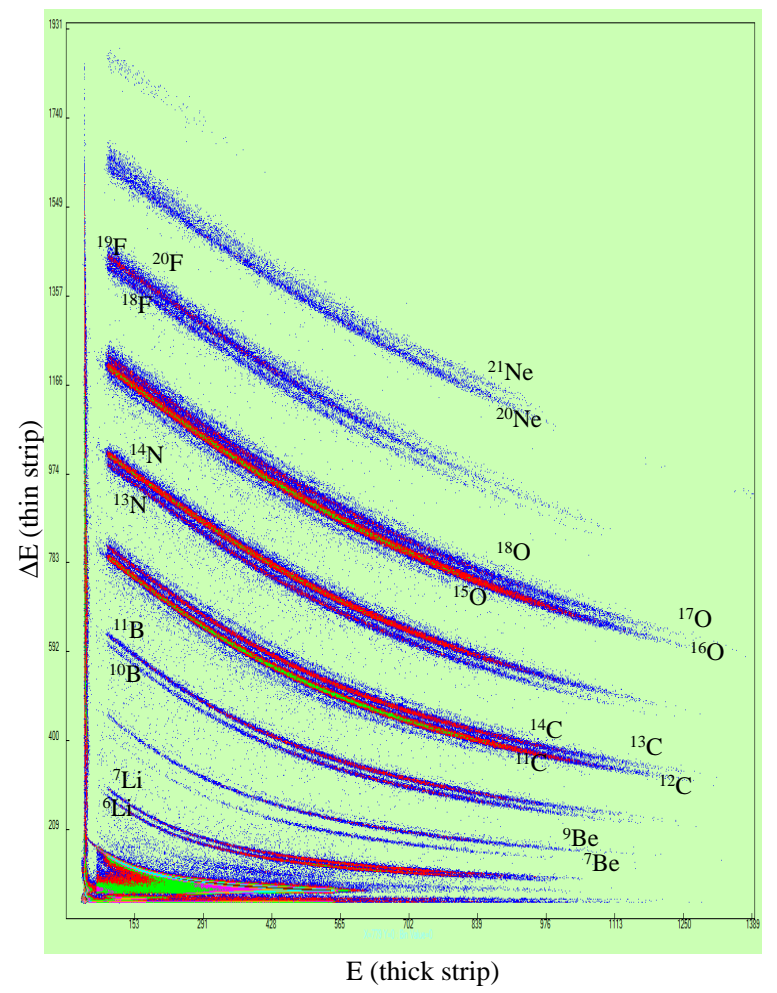

Figure 19: 2D Spectrum of intermediate mass fragments and their isotopes emitted in the reaction $193 \mathrm{MeV}^{20} \mathrm{Ne}$ on ${ }^{9} \mathrm{Be}$ obtained by a telescope.

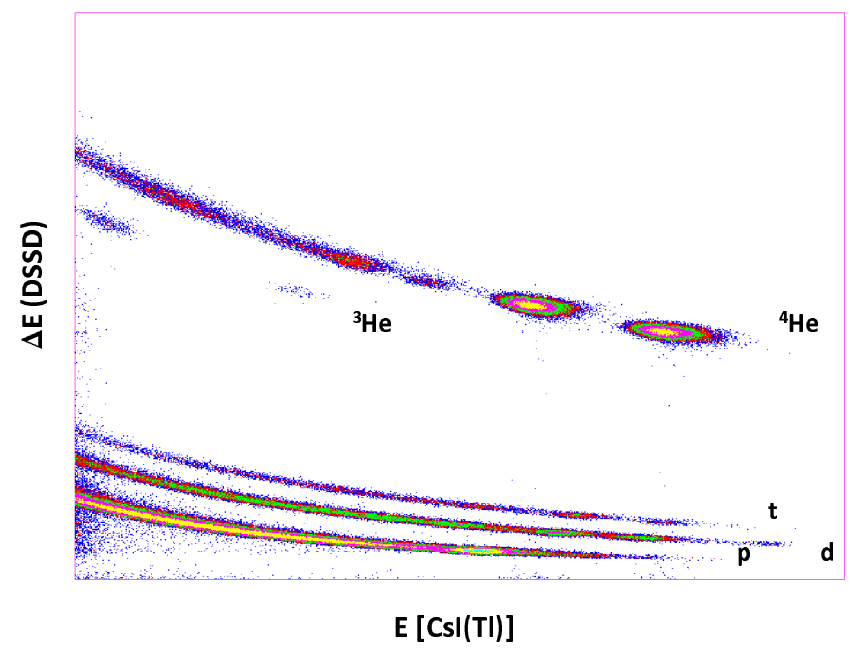

Figure 20: 2D Spectrum light charged particles emitted in the reaction $60 \mathrm{MeV} \alpha$ on ${ }^{12} \mathrm{C}$ obtained by a telescope.

tained by last two detectors of the 3-element telescope, i.e., DSSD and CsI(Tl) detector is shown in Fig. 20 13. It is seen that all isotopes of $\mathrm{Z}=1$ and 2 are clearly identified. These measurements clearly establish the expected level of performance of the array. The telescope is able to isotopically separate all fragments at least up to $\mathrm{Z}=10$, which was the bench mark of performance. In actual physics experiment, one encounters multiple hit events. Therefore, kinematic reconstruction of the events (identification of all particles, and their pixel positions and energies) is essential to extract the physics information. Here, a pixel corresponds the overlapping area between the orthogonal front and back side strips of the DSSD detector $\left(3 \times 3 \mathrm{~mm}^{2}\right.$ in the present case). A data reduction algorithm in ROOT framework [14] has been developed to identify the particles and reconstruct the events. The hit positions of the particles are identified by matching the energies deposited in the front and back strips, using a minimization routine specially developed for this purpose.

\subsection{Characterization of the backward part detectors}

Non-uniformity in doping of $\mathrm{CsI}(\mathrm{Tl})$ crystals causes nonuniformity in scintillation light output which affects the energy resolution. Non-uniformity has been measured in a similar way as mentioned Sec. 3.1.2, which has been found to be within 1\%. Energy resolution of detectors also depends on the variation of the thickness and microleak in the entrance window. The energy resolution of each detectors has been measured by using a $\alpha$ source of ${ }^{241} \mathrm{Am}$ having energy $5.486 \mathrm{MeV}$. In case of some of the detectors we found two peaks in the energy spectra from ${ }^{241} \mathrm{Am} \alpha$ source as shown in Fig. 21(a). After replacement of the mylar window, one peak disappeared as in Fig. 21(b). The extra peak was coming due to variation of thickness in the entrance mylar window. It was observed that the energy resolution of the detectors, depending on the volume of the crystal, varied between $\sim 4-6 \%$. In the 


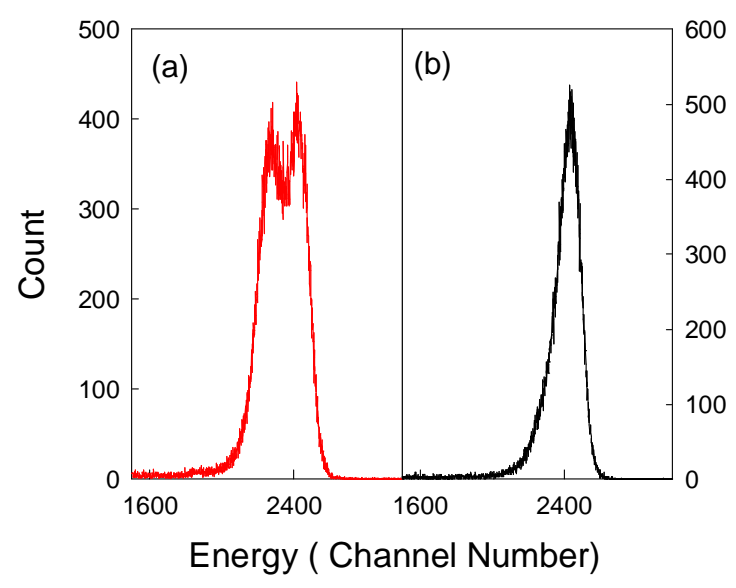

Figure 21: Energy spectra ${ }^{241} \mathrm{Am} \alpha$ source using $\mathrm{CsI}(\mathrm{Tl})$ detectors. The energy spectra of panel (a) was obtained from a detector having defective entrance window foil and panel (b) after replacement of the foil.

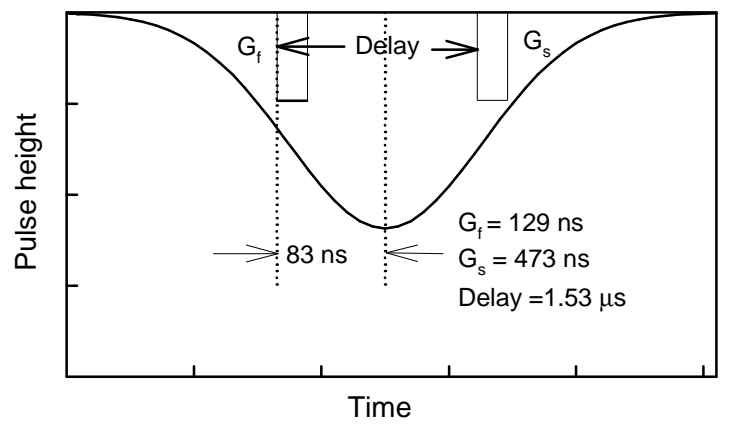

Figure 22: Schematic diagram of fast gate and slow gate.

backward array, light particles are identified by using the pulse shape discrimination (PSD) property of the CsI(Tl) detector. The light output of $\mathrm{CsI}(\mathrm{Tl})$ detectors have two components which can be approximately written as

$$
L(t)=L_{s} \exp \left(-t / \tau_{s}\right)+L_{f} \exp \left(-t / \tau_{f}\right)
$$

where $L(t)$ is the light output at time $t . \quad\left(L_{s}, \mathrm{~L}_{f}\right)$ and $\left(\tau_{s}, \tau_{f}\right)$ are amplitude and decay constant of scintillation light output of fast and slow components, respectively. $\tau_{f}$ depends on the stopping power $(d E / d x)$ of the particle in the $\operatorname{CsI}(\mathrm{Tl}): \tau_{f} \propto d E / d x$. This property is used to identify the particle using pulse shape discrimination (PSD) technique. Particle identification property of these detectors using PSD technique has been studied in-beam experiment using $50 \mathrm{MeV} \alpha$ beam from K130 cyclotron, VECC with ${ }^{27} \mathrm{Al}$ target. Here, charge integrations of the detector pulse were done over two gates, a fast gate $\left(G_{f}\right)$ and a slow gate $\left(G_{s}\right)$, as shown schematically in Fig 22, The spectrum obtained is shown in Fig. 23. It is seen that $p, d, t$ and $\alpha$ are clearly separated [15]. At low energy, the light output (L) of a $\mathrm{CsI}(\mathrm{Tl})$ detector depends nonlinearly on the deposited energy (E). Therefore, unlike Si detectors, the energy calibration of $\mathrm{CsI}(\mathrm{Tl})$ is not quite straightforward as it depends on particle type. Therefore,

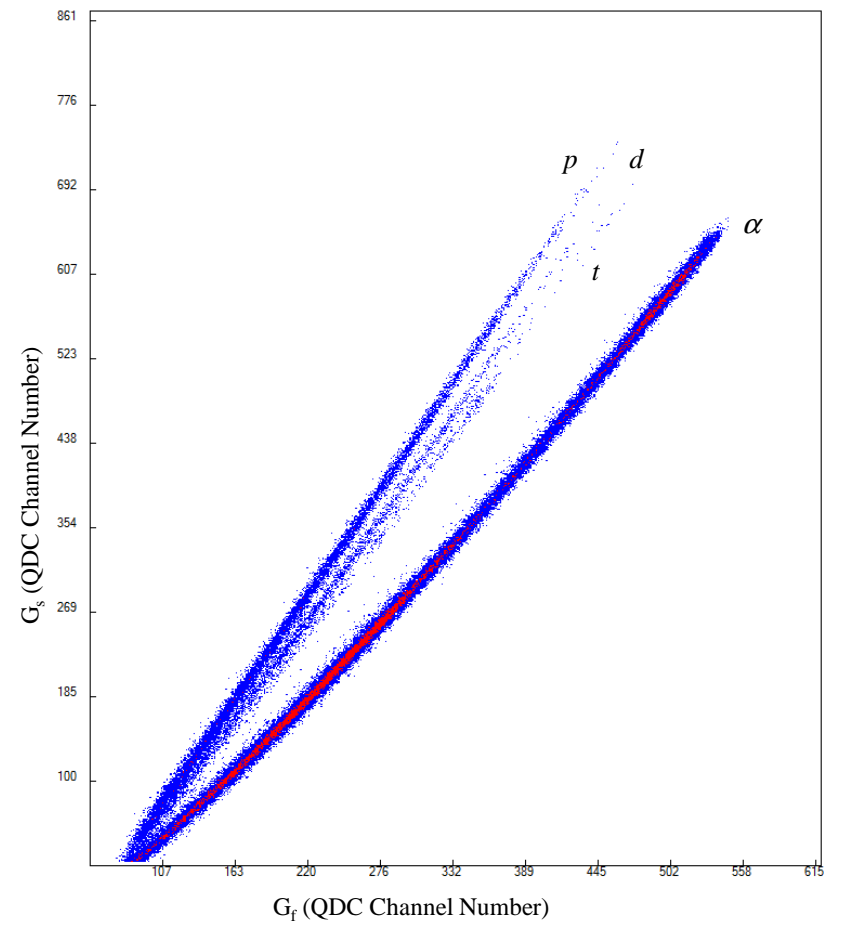

Figure 23: Fast gate - slow gate spectrum for gates and delay widths as in Fig. 22

Table 2: The fitted value of the parameters used in Eqn. 2] and 3

\begin{tabular}{ccccc}
\hline $\mathrm{Z}$ & $\mathrm{a}$ & $\mathrm{b}$ & $\mathrm{c}$ & $\mathrm{d}$ \\
\hline $\mathrm{p}$ & 0.0916 & 0.2412 & 0.0258 & - \\
$\alpha$ & 0.0941 & 0.7381 & 5.2631 & - \\
${ }^{7} \mathrm{Li}$ & 0.0994 & 4.9171 & 0.0773 & - \\
$\mathrm{p}, \alpha,{ }^{7} \mathrm{Li}$ & 0.0008 & 0.1040 & 0.0110 & 0.0455 \\
\hline
\end{tabular}

the energy calibration procedure for these detectors was tested using three types of particles, ${ }^{7} \mathrm{Li}, \alpha$ and protons. Elastically scattered ${ }^{7} \mathrm{Li}$ from a gold target as well as proton recoil peaks are used for this purpose. In addition, $\alpha$ and protons of known energies produced in the ${ }^{12} \mathrm{C}\left({ }^{12} \mathrm{C}\right.$, $\left.{ }^{4} \mathrm{He}\right){ }^{20} \mathrm{Ne}^{*}$ and ${ }^{12} \mathrm{C}\left({ }^{12} \mathrm{C}, p\right){ }^{23} \mathrm{Na}^{*}$ reactions at beam energy 40 and $25 \mathrm{MeV}$ feeding the excited states of $\mathrm{Ne}^{*}$ and ${ }^{23} \mathrm{Na}^{*}$ were used [16]. The light outputs were fitted separately as well as globally using two algorithms [16].

$$
E(L)=a L+b \ln (1+c L): \text { individual }
$$

and,

$$
E(L, Z, A)=a A Z^{2} L+\left(b+C A Z^{2}\right) L^{(1-d Z \sqrt{A})}: \text { global }
$$

Best fit parameter sets for both the algorithms are given in table 2

\subsection{Characterization of plastic phoswich detectors of ex- treme forward part}

Decay constants of the light output of these two plastics of the phoswich detector are different; for BC 408 (thin), 


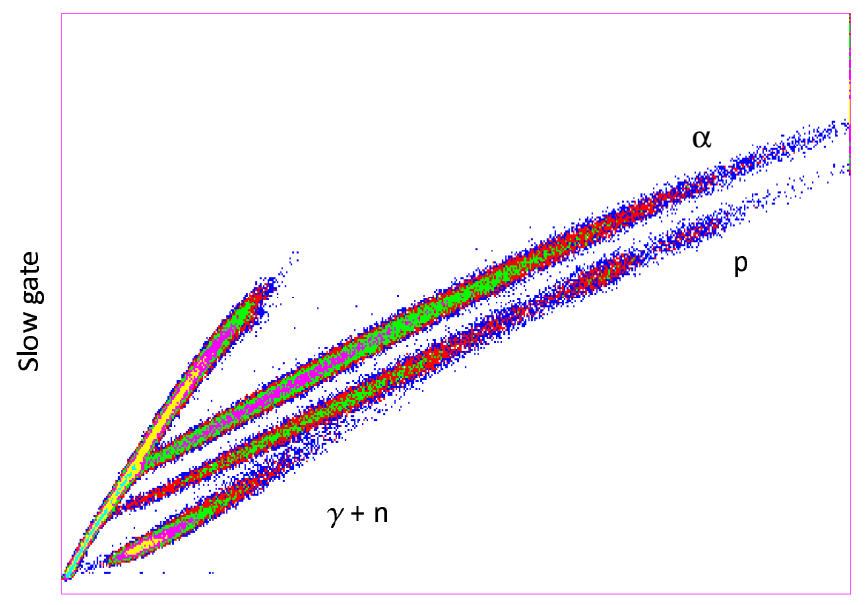

Fast gate

Figure 24: Fast gate - slow gate energy spectra obtained by plastic phoswich detector

the decay constant is 2.1 ns [10] and the same for BC 444 (thick) is 285 ns [10]. This thin-thick combination with fast-slow decay times acts as a $\Delta E-E$ combination. The charged particle incident on the detector produces light having fast and slow components which is collected by single photo multiplier tube (PMT). Two integration of charge, one fast gate and another slow gate, in proper time will give the light outputs proportional to energy deposited in thin and thick plastics separately and two dimensional plot of the two outputs provide information about the type and energy of the particle.

The performance of these detectors was tested in-beam using the reaction $145 \mathrm{MeV}{ }^{20} \mathrm{Ne}+{ }^{12} \mathrm{C}$. The PMT output signal was fed into fast amplifier and amplified output was divided into two parts. These two parts were then charge integrated using the two gates, each of width $60 \mathrm{~ns}$ and sepated by $240 \mathrm{~ns}$. A two dimensional plot of the two (Fig. 24) demonstrated the particle identification property of these detectors.

\subsection{Experiments using ChAKRA}

Experiment using full ChAKRA is planned with the beams from upcoming K500 superconducting cyclotron at VECC. For that purpose, a large, segmented, horizontal axis, reaction chamber (SHARC) 17] has already been developed to house the ChAKRA. In the meanwhile, different parts of the array, the segments of the forward array in particular, have been and are being extensively used in wide varieties of nuclear reaction studies at VECC and other accelerator laboratories across India (e.g., refs. 13], 18], [19, 20], [21], 22] ).

\section{Summary}

ChAKRA, the $4 \pi$ array of charged particle detectors have been developed at VECC for medium energy $(\lesssim$
$60 \mathrm{MeV} / \mathrm{A}$ ) reaction studies. However, part of ChAKRA can be used for the experiments at low $(\sim 10 \mathrm{MeV} / \mathrm{A})$ energy also. The array consisted of three independent blocks and each block is made up of different type of detector systems, depending on the nature of reaction products expected within the coverage of the particular block. Most important part of the ChAKRA is the high resolution forward array (angular coverage : $\theta \sim \pm 7^{0}-45^{0}$ ) which consists of 24 charged particle telescope each of which is made by three layers of detectors [single side silicon $\operatorname{strip}(\Delta \mathrm{E})$ + double side silicon strip $(\mathrm{E} / \Delta \mathrm{E})+\mathrm{CsI}(\mathrm{Tl})(\mathrm{E})]$. This part is the main workhorse of the array which can identify isotopes of light charged particle as well as heavy charged particles at least up to $\mathrm{Z}=10$. Backward part of the array consists of $112 \mathrm{CsI}(\mathrm{Tl})$ detectors. It covers an angular range of $\theta \sim \pm 45^{0}-175^{\circ}$. Pulse shape discrimination method is used for these detectors to detect light charged particles with their isotopic separation. Extreme forward part of the array consists of 32 plastic phoswich detectors, covering the most forward angular range of $\theta \sim \pm 3^{0}-7^{0}$. These fast detectors are capable to detect light as well as heavy charged particles and handle high count rates. The whole array was made in several detachable segments in order that any part of the array can be used separately or in conjunction with other detector systems as and when need arises. Part of ChAKRA has already been successfully used in several experiments at low energy $(\lesssim 10 \mathrm{MeV} / \mathrm{A}$. It is hoped that, with the advent of upcoming new accelerators (superconducting cyclotron, rare ion beam facility) at VECC, the ChAKRA will be immensely useful for next generation nuclear reaction studies at VECC.

\section{References}

\section{References}

[1] J. Pouthas, B. Borderie, R. Dayras, E. Plagnol, M. Rivet, F. Saint-Laurent, J. Steckmeyer, G. Auger, C. Bacri, S. Barbey, A. Barbier, A. Benkirane, J. Benlliure, B. Berthier, E. Bougamont, P. Bourgault, P. Box, R. Bzyl, B. Cahan, Y. Cassagnou, D. Charlet, J. Charvet, A. Chbihi, T. Clerc, N. Copinet, D. Cussol, M. Engrand, J. Gautier, Y. Huguet, O. Jouniaux, J. Laville, P. L. Botlan, A. Leconte, R. Legrain, P. Lelong, M. L. Guay, L. Martina, C. Mazur, P. Mosrin, L. Olivier, J. Passerieux, S. Pierre, B. Piquet, E. Plaige, E. Pollacco, B. Raine, A. Richard, J. Ropert, C. Spitaels, L. Stab, D. Sznajderman, L. Tassan-got, J. Tillier, M. Tripon, P. Vallerand, C. Volant, P. Volkov, J. Wieleczko, G. Wittwer, Indra, a 4pi charged product detection array at ganil Nuclear Instruments and Methods in Physics Research Section A: Accelerators, Spectrometers, Detectors and Associated Equipment 357 (2) (1995) 418 - 442. doi:https://doi.org/10.1016/0168-9002(94)01543-0

URL http://www.sciencedirect.com/science/article/pii/0168900294

[2] M. Wallace, M. Famiano, M.-J. van Goethem, A. Rogers, W. Lynch, J. Clifford, F. Delaunay, J. Lee, S. Labostov, M. Mocko, L. Morris, A. Moroni, B. Nett, D. Oostdyk, R. Krishnasamy, M. Tsang, R. de Souza, S. Hudan, L. Sobotka, R. Charity, J. Elson, G. Engel, The high resolution array (hira) for rare isotope beam experiments, Nuclear Instruments and Methods in Physics Research Section A: Accelerators, Spectrometers, Detectors 
and Associated Equipment 583 (2) (2007) 302 - 312. doi:https://doi.org/10.1016/j.nima.2007.08.248

D. Pandit, S. R. Banerjee, A. Roy, P. Dhara, URL http://www.sciencedirect.com/science/article/pii/S0168900207061547X Rev. $\quad$ C 78 (2008)

[3] S. Aiello, A. Anzalone, Baldo, M. Cardella, G. Cavallaroo, E. Filippo, S. Pietro, A.Di ans Feminoc, P. Figuera, P. Guazzonid, C. Iacono-Manno, G. Lanzanob, U. Lombardo, S. Nigro, A. Muumarra, A. Pagano, M. Papab, S. Pirroneb, G. Politi, F. Porto, A. Rapisardab, F. Rizzo, S. Sambatarob, M. Sperduto, C. Suterah, L. Zettad, Chimera: a project of a $4 \pi$ detector for heavy ion reactions studies at intermediate energy, Nucl. Inst. Meth. AS83 ((1995)) 461.

[4] B. Davin, R. de Souza, R. Yanez, Y. Larochelle, R. Alfaro, H. Xu, A. Alexander, K. Bastin, L. Beaulieu, J. Dorsett, G. Fleener, L. Gelovani, T. Lefort, J. Poehlman, R. Charity, L. Sobotka, J. Elson, A. Wagner, T. Liu, X. Liu, W. Lynch, L. Morris, R. Shomin, W. Tan, M. Tsang, G. Verde, J. Yurkon, Lassa: a large area silicon strip array for isotopic identification Nuclear Instruments and Methods in Physics Research Section A: Accelerators, Spectrometers, Detectors and Associated Equipment 473 (3) (2001) 302 - 318. doi:https://doi.org/10.1016/S0168-9002(01)00295-9

doi : 10.1103/PhysRevC.78.027602

URL https://link.aps.org/doi/10.1103/PhysRevC.78.027602

[14] https://root.cern.ch/

[15] S. Kundu, C. Bhattacharya, T. K. Rana, K. Banerjee, S. Bhattacharya, J. K. Meena, R. Saha, G. Mukherjee, T. K. Ghosh, R. Pandey, P. Roy, M. Gohil, V. Srivastava, A. Dey, G. Pal, S. Roy, S. R. Bajirao, C. Nandi, Charged particle detector array: $45^{0}-175^{0 *}$ DAE-BRNS Symp 57 (2012) 864-865.

URL www . sympnp.org/proceedings

[16] S. Kundu, K. Banerjee, T. Rana, C. Bhattacharya, P. C. Rout, A. Mitra, E. Mirgule, V. Nanal, S. Kumar, V. Datar, D. Chakrabarty, S. Bhattacharya, geA.paDtey,es( . Mukherjee, T. Ghosh, D. Gupta, J. Meena, Testing of a csi (tl) detector and its energy calibration DAEBRNS Symp 51 (2006) 618-619.

URL http://www . sympnp.org/ 17] S. Kundu, S. Bhattacharya, J. K. Meena, T. K. Ghosh URL http://www.sciencedirect.com/science/article/pii/S016890020100298ttacharjee, P. Mukhopadhyay, C. Bhattacharya,

[5] The FAZIA Collaboration, R. Bougault, G. Poggi, S. Barlini, B. Borderie, G. Casini, A. Chbihi, N. Le Neindre, M. Pârlog, G. Pasquali, S. Piantelli, Z. Sosin, G. Ademard, R. Alba, A. Anastasio, S. Barbey, L. Bardelli, M. Bini, A. Boiano, M. Boisjoli, E. Bonnet, R. Borcea, B. Bougard, G. Brulin, M. Bruno, S. Carboni, C. Cassese, F. Cassese, M. Cinausero, L. Ciolacu, I. Cruceru, M. Cruceru, B. D'Aquino, B. De Fazio, M. Degerlier, P. Desrues, P. Di Meo, J. A. Dueñas, P. Edelbruck, S. Energico, M. Falorsi, J. D. Frankland, E. Galichet, K. Gasior, F. Gramegna, R. Giordano, D. Gruyer, A. Grzeszczuk, M. Guerzoni, H. Hamrita, C. Huss, M. Kajetanowicz, K. Korcyl, A. Kordyasz, T. Kozik, P. Kulig, L. Lavergne, E. Legouée, O. Lopez, J. Łukasik, C. Maiolino, T. Marchi, P. Marini, I. Martel, V. Masone, A. Meoli, Y. Merrer, L. Morelli, F. Negoita, A. Olmi, A. Ordine, G. Paduano, C. Pain, M. Pałka, G. Passeggio, G. Pastore, P. Pawłowski, M. Petcu, H. Petrascu, E. Piasecki, G. Pontoriere, E. Rauly, M. F. Rivet, R. Rocco, E. Rosato, L. Roscilli, E. Scarlini, F. Salomon, D. Santonocito, V. Seredov, S. Serra, D. Sierpowski, G. Spadaccini, C. Spitaels, A. A. Stefanini, G. Tobia, G. Tortone, T. Twaróg, S. Valdré, A. Vanzanella, E. Vanzanella, E. Vient, M. Vigilante, G. Vitiello, E. Wanlin, A. Wieloch, W. Zipper, The fazia project in europe: R\&d phase, The European Physical Journal A 50 (2) (2014) 47. doi:10.1140/epja/i2014-14047-4 URL https : //doi .org/10.1140/epja/i2014-14047-4

[6] http://www.micronsemiconductor.co.uk

[7] http://www.scionix.nl/

[8] LISE ++, http://lise.nscl.msu.edu/lise.html

[9] D. Guinet, B. Chambon, B. Cheynis, A. Demeyer, D. Drain, X. Hu, C. Pastor, L. Vagneron, K. Zaid, A. Giorni, D. Heuer, A. Lleres, J. Viano,

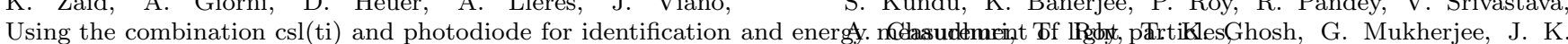
Nuclear Instruments and Methods in Physics Re- Meena, S. K. Pandit, K. Mahata, A. Shrivastava, V. Nanal, search Section A: Accelerators, Spectrometers, Detectors and Associated Equipment 278 (2) (1989) 614 - 616. doi:https://doi.org/10.1016/0168-9002(89)90889-9 T. K. Rana, K. Banerjee, G. Mukherjee, S. R. Banerjee, D. L. Bandyopadhyay, M. Ahammed, P. Bhattacharya, A large high vacuum reaction chamber for nuclear physics research at ve Journal of Physics: Conference Series 390 (1) (2012) 012075. URL http://stacks .iop.org/1742-6596/390/i=1/a=012075

[18] T. K. Rana, S. Bhattacharya, C. Bhattacharya, S. Kundu, K. Banerjee, T. K. Ghosh, G. Mukherjee, R. Pandey, P. Roy, V. Srivastava, M. Gohil, J. K. Meena, H. Pai, A. K. Saha, J. K. Sahoo, R. M. Saha, Estimation of direct components of the decay of the hoyle state $\begin{array}{lllll}\text { Phys. Rev. } & \text { C } & 88 & \text { (2013) } & 021601 .\end{array}$ doi:10.1103/PhysRevC.88.021601

URL https://link.aps.org/doi/10.1103/PhysRevC.88.021601

[19] V. Srivastava, C. Bhattacharya, T. K. Rana, S. Manna, S. Kundu, S. Bhattacharya, K. Banerjee, P. Roy, R. Pandey, G. Mukherjee, T. K. Ghosh, J. K. Meena, T. Roy, A. Chaudhuri, M. Sinha, A. Saha, M. A. Asgar, A. Dey, S. Roy, M. M. Shaikh, Experimental study of ${ }^{26} \mathrm{Al}$ through the $1 n$ pick-up reaction ${ }^{27} \mathrm{Al}(d, t)$ $\begin{array}{lllll}\text { Phys. Rev. } & \text { C } & 91 & \text { (2015) } & 054611 .\end{array}$ doi:10.1103/PhysRevC.91.054611

URL https://link.aps.org/doi/10.1103/PhysRevC.91.054611

[20] V. Srivastava, C. Bhattacharya, T. K. Rana, S. Manna, S. Kundu, S. Bhattacharya, K. Banerjee, P. Roy, R. Pandey, G. Mukherjee, T. K. Ghosh, J. K. Meena, T. Roy, A. Chaudhuri, M. Sinha, A. K. Saha, M. A. Asgar, A. Dey, S. Roy, M. M. Shaikh, Experimental investigation of $t=1$ analog states of ${ }^{26} \mathrm{Al}$ and ${ }^{26} \mathrm{Mg}$, $\begin{array}{llllll}\text { Phys. Rev. } & \text { C } & 93 & \text { (2016) } & 044601 .\end{array}$ doi:10.1103/PhysRevC.93.044601

URL https://link.aps.org/doi/10.1103/PhysRevC.93.044601

21] S. Manna, T. K. Rana, C. Bhattacharya, S. Bhattacharya, S. Kundu, K. Banerjee, P. Roy, R. Pandey, V. Srivastava, Survival of cluster correlation in dissipative binary breakup of ${ }^{24,25} \mathrm{Mg}^{*}$ $\begin{array}{lllll}\text { Phys. Rev. } & \text { C } & 94 & \text { (2016) } & 051601 .\end{array}$ doi:10.1103/PhysRevC.94.051601 URLhttp://www.sciencedirect.com/science/article/pii/0168900289d8B(19ttps://link.aps.org/doi/10.1103/PhysRevC.94.051601

[10] https://www.crystals.saint-gobain.com/

[11] https://www.mesytec.com

[12] S. Manna, C. Bhattacharya, T. Rana, S. Kundu, R. Pandey, A. Sen, D. Paul, P. Roy, T. Ghosh, G. Mukherjee, S. Mukhopadhyay, S. Nandi, J. Meena, R. Saha, A. Saha, J. Sahoo, S. Dalal, Cluster emission studies in 28,29si* DAE-BRNS Symp 63 (2018) 678-679.

URL www.sympnp.org/proceedings

[13] T. K. Rana, C. Bhattacharya, S. Kundu, K. Banerjee, S. Bhattacharya, A. Dey, T. K. Ghosh, G. Mukherjee, J. K. Meena, D. Gupta, S. Mukhopadhyay,

[22] R. Pandey, S. Kundu, C. Bhattacharya, K. Banerjee, T. K. Rana, S. Manna, G. Mukherjee, J. K. Meena, A. Chaudhuri, T. Roy, P. Roy, M. A. Asgar, V. Srivastava, A. Dey, M. Sinha, T. K. Ghosh, S. Bhattacharya, S. K. Pandit, K. Mahata, P. Patle, S. Pal, A. Shrivastava, V. Nanal, Fragment emission mechanism in the ${ }^{32} \mathrm{~S}+{ }^{12} \mathrm{C}$ reaction, $\begin{array}{lllll}\text { Phys. Rev. } & \text { C } & 95 & \text { (2017) } & \end{array}$ doi:10.1103/PhysRevC.95.064603

URL https://link.aps.org/doi/10.1103/PhysRevC.95.064603 